grey literature searches, and (4) eligibility biases, such as those based on the language of publication or on particular indicators of quality, which may result in the exclusion of non-English reports and small-scale studies from regions with less developed health research infrastructure. Conclusion A systematic review must set strict inclusion criteria, but the process used to select eligible studies may introduce a wide variety of biases. Awareness of the factors that restrict the comprehensiveness of systematic reviews will allow researchers to weigh and address these limitations. Improved systematic reviews will form a stronger foundation for evidence-based policy.

\section{P1-183 INFLUENZA VACCINATION IN PROSTATE CANCER SURVIVORS IN THE ELECTRONIC HEALTH RECORD ERA}

doi:10.1136/jech.2011.142976d.76

S J Jacobsen, * J M Slezak, L P Wallner, R Haque, H F Tseng, R K Loo, V P Quinn. Kaiser Permanente Southern California, Pasadena, California, USA

Introduction While most men diagnosed with prostate cancer die from causes other than their cancer, many think that subsequent care focuses on their prostate cancer-related issues. We investigated whether influenza and pneumococcal vaccination practices were diminished in a cohort of men following their diagnosis of prostate cancer, in an environment that has implanted an electronic health record with multiple redundant reminders.

Methods We used information collected as part of the California Mens Health Study, a prospective cohort study of nearly 40000 men ages 45-69 years at baseline in 2002 who were recruited through the Kaiser Permanente Southern California Health Plan. We identified all 1636 men who were newly diagnosed with prostate cancer from 2002 through 2008 and examined the use of influenza vaccine in the year prior to and subsequent to their cancer diagnosis, in the period before and after the introduction of the electronic health record.

Results Of the 973 and 663 men with prostate cancer in the pre- and post-electronic era, 312 (33\%) and 391 (59\%) had an influenza vaccine in the year prior to diagnosis compared to 374 (39\%) and 406 (61\%) in the years following (Matched OR (95\% CI) 4.84 (3.62 to $6.47)$ and 1.64 (1.14 to 2.36)). Similar results were obtained when expanded to 2 years.

Conclusion These data suggest that once diagnosed with prostate cancer, no less attention is paid to preventive care as measured by influenza vaccination. Moreover, even with the dramatic increases in vaccination rates associated with the electronic record, there remained a significant improvement following cancer diagnosis.

\section{P1-184 A META-ANALYSIS OF THE PREVALENCE OF HIV/SYPHILIS AMONG CHINESE BISEXUAL MEN WHO HAVE SEX WITH MEN (MSM)}

doi:10.1136/jech.2011.142976d.77

${ }^{1} \mathrm{X}$ Jun-Jie, ${ }^{*}{ }^{1} \mathrm{Y} \mathrm{Ke},{ }^{2} \mathrm{~K}$ H Reilly, ${ }^{1} \mathrm{Z}$ Jing, ${ }^{1} \mathrm{~J}$ Yong-Jun, ${ }^{1} \mathrm{~S}$ Hong. ${ }^{1}$ The Key Laboratory of AIDS Immunology of Ministry of Health, No 1 Hospital of China Medical University, Shenyang, China; ${ }^{2}$ National Center for AIDS/STD Control and Prevention, Chinese Center for Disease Control and Prevention (CDC), Beijing, P.R. China

Introduction In China, sexual transmission has become the major route of HIV transmission, prevalence of HIV is rapidly increasing among China men who have sex with men (MSM). This study investigate the bisexual behaviour among MSM and explore their $\mathrm{HIV} /$ syphilis prevalences stratified by sexual behaviour in order to inform the development of targeted preventive strategies.

Methods The Pubmed, Chinese Biomedical (CBM), Chinese National Knowledge Infrastructure (CNKI), VIP, Wanfang database, and Google Scholar databases were searched through June 2010 to identify relevant articles. Data of eligible citations was extracted by two reviewers. All analyses were performed with Stata 10.0.

Results Fifty studies (including 28739 total MSM subjects) met the selection criteria. Aggregated findings indicated that the estimated prevalence of bisexual behaviour among MSM in China is $31.2 \%$ (95\% CI $28.1 \%$ to $34.5 \%$ ). HIV and syphilis prevalences were $5.4 \%$ and $11.4 \%$, respectively among MSM engaging in bisexual behaviour and $3.8 \%$ and $9.3 \%$ among MSM only having sex with men, in which HIV prevalence among MSM engaging in sex with both men and women was significantly higher than those who only have sex with men (OR 1.30; 95\% CI 1.04 to $1.62 ; \mathrm{p}=0.02$ ).

Conclusion There is a high prevalence of bisexual behaviour among men who have sex with men in China, and bisexual behaviour is significantly associated with increased HIV infection risk. The results of this meta-analysis highlight a critical pattern of HIV transmission among MSM in China and indicate that targeted interventions aimed at encouraging safe sex practices and promoting societal and family acceptance of MSM are urgently needed.

\section{P1-185 ESTIMATING THE ATTRIBUTABLE BURDEN OF CARDIOVASCULAR DISEASE FOLLOWING REDUCING THE PREVALENCE OF DIABETES TO THEORETICAL AND FEASIBLE MINIMUM RISK LEVEL}

doi:10.1136/jech.2011.142976d.78

${ }^{1} \mathrm{M}$ Karami, ${ }^{*}{ }^{1,2} \mathrm{D}$ Khalili, ${ }^{3} \mathrm{~B}$ Eshrati. ${ }^{1}$ Shahid Beheshti University of Medical Sciences, Tehran, Iran; ${ }^{2}$ Research Institute for Endocrine Sciences, Tehran, Iran; ${ }^{3}$ Arak University of Medical Sciences, Arak, Iran

Introduction Knowledge of the magnitude of attributable burden to cardiovascular disease (CVD) by diabetes, especially based on updated data are needed for health policy, priority setting and preventing CVD. This study aimed to estimate the contribution of diabetes to the attributable burden of cardiovascular disease in Iran. Methods WHO Comparative Risk Assessment (CRA) methodology was used to calculating Potential Impact Fraction (PIF). Data on the Prevalence of newly diagnosed diabetes mellitus (NDM) and known diabetes mellitus (KDM) were obtained from $3^{\text {rd }}$ Iranian surveillance of risk factors of non- communicable diseases and data on corresponding measures of effect were derived from a nationalspecific study with age and multivariate adjusted HRs. PIF were estimated on both theoretical minimum and feasible minimum risk. Uncertainty for the attributable burden was estimated by Monte Carlo simulation-modelling techniques incorporating sources of uncertainty.

Results Based on multivariate- adjusted HRs, by reducing the per cent of Iranian women with diabetes from $10.05 \%$ to the feasible minimum risk level that is, $5 \%, 6.8 \%$ (95\% uncertainty intervals: 3.5 to 9.8 ) of attributable Disability Adjusted Life Years (DALYs) to CVD are avoidable and the corresponding value for men were $3.1 \%$ (95\% uncertainty intervals: 1.4 to 4.8 ).

Conclusion Although data on the Prevalence of diabetes and corresponding measures of effect were obtained from an updated and country- specific source, but to better priority setting, PIF should be applied to updated and revised burden of CVD.

\section{P1-186 CHALLENGES IN NATURAL EXPERIMENT RESEARCH: POTENTIAL LESSONS FROM THE H1N1 PANDEMIC}

doi:10.1136/jech.2011.142976d.79

${ }^{1,2} \mathrm{~S}$ V Katikireddi, ${ }^{1} \mathrm{~K}$ Skivington, ${ }^{1} \mathrm{M}$ Egan, ${ }^{2} \mathrm{D}$ G Mackenzie, ${ }^{1} \mathrm{~L}$ Bond. ${ }^{1} \mathrm{MRC}$ Social and Public Health Sciences Unit, Glasgow, UK; ${ }^{2}$ NHS Lothian, Edinburgh, UK

Introduction Research on natural experiments (NEs) allows investigation into topics that are important for policy-makers, including 
many social determinants of health. NEs can be conceptualised as differing from other research in three important ways. First, unlike trials, investigators cannot allocate the exposure of interest which is instead externally decided. Second, researchers are unable to influence the exposure's characteristics which results in uncertainty of the fidelity, dose and whether the NE will occur at all. Third, and in contrast to many observational studies, the timing of the exposure and hence research itself, cannot be chosen by researchers.

Methods Using three case studies of research on H1N1 (two quantitative sero-epidemiology studies, one qualitative study) we identified key factors that allowed us to successfully conduct our investigation of a NE. We compared these factors with the wider NE literature to identify common barriers and facilitators to research. Results We identified key external factors that influence capacity to successfully take advantage of NEs. As a result of the uncertainty of a NE occurring and the rapid response required, flexibility is needed by researchers, policymakers, practitioners, ethics committees and funders. This is achievable when research is perceived as immediately important for health (as in H1N1) but may otherwise be difficult.

Discussion We suggest researchers investigating NEs face additional challenges to traditional observational studies. While some barriers are insurmountable, actions such as rapid response funding, fasttrack ethics procedures and improvements in routine data can create a more conducive environment allowing policy-relevant evaluation

\section{P1-187 ACCESS TO ALCOHOL OUTLETS AND HARMFUL ALCOHOL CONSUMPTION: A MULTILEVEL STUDY IN MELBOURNE, AUSTRALIA}

doi:10.1136/jech.2011.142976d.80

${ }^{1} \mathrm{~A}$ Kavanagh, ${ }^{2} \mathrm{M}$ Kelly, ${ }^{1} \mathrm{~L}$ Krnjacki, ${ }^{3} \mathrm{~L}$ Thornton, ${ }^{4} \mathrm{D}$ Jolley, ${ }^{5} \mathrm{~S} \vee$ Subramanian, ${ }^{6} \mathrm{G}$ Turrell, ${ }^{1} \mathrm{R}$ Bentley. ${ }^{1}$ The University of Melbourne, Victoria, Australia; ${ }^{2}$ La Trobe University, Victoria, Australia; ${ }^{3}$ Deakin University, Victoria, Australia; ${ }^{4}$ Monash University, Victoria, Australia; ${ }^{5}$ Harvard University, Boston, USA; ${ }^{6}$ Oueensland University of Technology, Queensland, Australia

Introduction While developed countries have either introduced, or are considering, legislation to restrict the number of alcohol outlets, there is little evidence to support this strategy except in the USA.

Methods Multilevel study of 2334 adults aged 18 to 75 years from 49 census collector districts (the smallest spatial unit in Australia at the time of survey) in Melbourne, Australia. Alcohol outlet density was defined as the number of outlets within a one kilometre road network of respondents' homes and proximity was the shortest road network distance to the closest outlet from their home. Using multilevel logistic regression we estimated the association between outlet density and proximity and four measures of harmful alcohol consumption: drinking at levels associated short-term harm at least weekly and monthly; drinking at levels associated with long-term harm and frequency of consumption.

Results Density of outlets was associated with increased risk of alcohol related harm with the strongest association evident for drinking at levels associated with short term harm at least weekly (OR 1.10, 95\% CI 1.04 to 1.16). When density was fitted as a categorical variable, the highest risk of drinking at levels associated with short-term harm was when there were eight or more outlets (short-term harm weekly: OR 2.36, 95\% CI 1.22 to 4.54 and shortterm harm monthly: OR $1.80,95 \%$ CI 1.07 to 3.04$)$. We found no evidence to support an association between proximity and harmful alcohol consumption.

Conclusion Restricting the number of off-premise alcohol outlets is likely to reduce levels of harmful alcohol consumption.

\section{P1-188 ANALYSIS OF QUESTIONNAIRE SURVEYS ON MOTHERS' ANXIETIES ABOUT CHILD-REARING IN RURAL AREAS OF JAPAN}

doi:10.1136/jech.2011.142976d.81

${ }^{1} \mathrm{H}$ Kawasaki, ${ }^{2} \mathrm{M}$ Nlshiyama, ${ }^{3} \mathrm{M}$ Fujita, ${ }^{3} \mathrm{P}$ D'Angelo, ${ }^{1} \mathrm{~S}$ Moriwaki. ${ }^{1}$ Hiroshima University, Hiroshima, Japan; 'Hiroshima Bunkyo Women's University, Hiroshima, Japan; ${ }^{3}$ Doi Clinic, Hiroshima, Japan

Background In Japan, as society changes, becoming ever more isolated and with rises in nuclear families, mothers are increasingly concerned and uneasy about child care. These changes have also coincided with a significant decrease in birth rates. The government took out the "indicator of support plan for the 21st century" in 2001. So, each prefecture had to draw up a concrete plan by 2005 .

Purpose The purpose of study was to explore factors affecting mothers' anxieties and to evaluate effects of a child care support plan. Sample and Statistical Analysis The questionnaire surveys were conducted with 1000 mothers, selected randomly, in 2002 and 2008. Mothers' anxiety was measured using feelings of loneliness, confusion about child-rearing and lack of standards as the children's development process. These were analysed using "ordered categorical logistic regression". The explanatory variables were mother's age, child's age and child-rearing methods.

Results 'Feelings of loneliness' was negatively correlated with a child's age ( $p$ value $=0.07$ ) and a maternal age $(p$ value $=0.044$ ) Mothers' mental instability and a lack of families' cooperation significantly related with 'feelings of loneliness' ( $p$ values $=0.000$, 0.000 , respectively). "Feelings of loneliness" and "confusion about child-rearing" in 2008 (after implementation of the child care support plan) were reduced compared with those in 2002 ( $p$ values $=0.069,0.065$, respectively).

Conclusions The introduction of a child care support plan including opportunities for counselling, friendship and activities increased family co-operation and more social interaction for both mother and child. The research shows these can be effective methods in reducing mothers' anxieties.

\section{P1-189 NET BENEFIT FRACTION AS A MODIFIED POPULATION ATTRIBUTABLE FRACTION TO EVALUATE THE CLINICAL PERFORMANCE OF A RISK FUNCTION}

doi:10.1136/jech.2011.142976d.82

${ }^{1,2} \mathrm{D}$ Khalili, ${ }^{*}{ }^{1} Y$ Mehrabi, ${ }^{2} \mathrm{M}$ Bozorgmanesh. ${ }^{1}$ Department of Epidemiology, School of public health, Shahid Beheshti University of Medical Sciences, Tehran, Iran; ${ }^{2}$ Prevention of Metabolic Disorders Research Center, Research Institute for Endocrine Sciences, Shahid Beheshti University of Medical Sciences, Tehran, Iran

Introduction Net benefit (NB) has been suggested to verify the clinical usefulness of a model beyond its discrimination and calibration but it has a complex meaning in prediction models. Here we define net benefit fraction (NBF) using Population Attributable Fraction (PAF) index.

Methods According to Vickers and Elkin (2006), in models that predict diseases to treat high risk individuals, NB is defined as true positive (TP) rate minus weighted false positive (wFP) rate; the weight is the odds at the threshold probability for treatment $(\mathrm{Pt} /[1$ $\mathrm{Pt}]$ ). Dividing NB by its maximum, incidence of disease, gives the portion of incidence prevented by the treatment. A shortcoming of $\mathrm{NB}$ is that it assumes the treatment reduces the incidence in TPs to zero. On the other hand, PAF is defined as reduction of the risk to below the threshold, that is, to that of low risk population. However, PAF does not take into account the false positives. Therefore, we suggest NBF calculated as PAF minus wFP rate, the latter divided by incidence. We applied the method to calculate the clinical performance of Framingham risk function at routine 